\title{
ultrastrutal investigation on Pseudo Chediak-Higashi abnormality in acute lymphoblastic leukemia: A case report
}

\author{
Jing Liu ${ }^{1}$, Shu-xu Dong ${ }^{1}$, Ying $\mathrm{Li}^{2}$, Yi-dan $\mathrm{Xu}^{1}$, and Yong-xin $\mathrm{Ru}^{3}$ \\ ${ }^{1}$ State Key Laboratory of Experimental Hematology, National Clinical Research Center for \\ Blood Diseases, Institute of Hematology Blood Diseases Hospital, Chinese Academy of \\ Medical Sciences Peking Union Medical College \\ ${ }^{2}$ Tianjin Neurological Institute, Tianjin Medical University General Hospital \\ ${ }^{3}$ Chinese Academy of Medical Sciences and Peking Union Medical College State Key \\ Laboratory of Experimental Hematology
}

September 24, 2021

\begin{abstract}
Pseudo Chediak-Higashi $(\mathrm{PCH})$ granules are mainly common in acute myeloid leukemia. Here we found a patient diagnosed with common-B acute lymphoblastic leukemia(ALL) with PCH cytoplasmic granules, which was rarely seen in daily diagnosis. The morphology of the granules is different in transmission electron microscope (TEM) from cases reported before. There is a vesicle with various-sized particles surrounding the nucleus, in addition to the particles, a multiple-layer membrane structure was also detected in the vesicle.
\end{abstract}

Ultrastructural investigation on Pseudo Chediak-Higashi abnormality in acute lymphoblastic leukemia: A case report

Jing Liu ·, Shu-xu Dong; ,Ying Li , Yi-dan Xu, Yong-xin Ru

\section{Abstract}

Pseudo Chediak-Higashi $(\mathrm{PCH})$ granules are mainly common in acute myeloid leukemia. Here we found a patient diagnosed with common-B acute lymphoblastic leukemia(ALL) with PCH cytoplasmic granules, which was rarely seen in daily diagnosis. The morphology of the granules is different in transmission electron microscope (TEM) from cases reported before. There is a vesicle with various-sized particles surrounding the nucleus, in addition to the particles, a multiple-layer membrane structure was also detected in the vesicle.

\section{Introduction}

Pseudo-Chediak-Higashi (PCH) anomaly is seen in various types of leukemia,including acute monoblastic leukemia $\left(\mathrm{AML}_{5} \mathrm{M}_{5}{ }^{1}\right.$, mixed phenotype acute leukemia ${ }^{2}$, acute myeloid leukemia ${ }^{3},{ }^{4}$,chronic myeloid leukemia $(\mathrm{CML})^{5}$, and myelodysplastic syndrome(MDS $)^{6}$.PCH is characterized by the appearance of eosinophilic granules of different sizes in cytoplasm of abnormal leukocytes when examined by light microscope. The disease shares similarities in appearance with the granules noted in Chediak-Higashi syndrome(CHS).In some rare cases, intracytoplasmic vacuoles are positive for myeloperoxidase(MPO) and periodic acid-Schiff (PAS) around the myeloblasts, which probably result from the accumulation of metabolic waste or damaged organelles ${ }^{3}$.Although there are some reports on the abnormality of cells in acute lymphoblastic leukemia(ALL $)^{7-9}$, the abnormal granules have been rarely interpreted. Herein, we report a rare case of ALL in a young patient because of rare morphology. 


\section{Case description}

A 9-year-old girlwas admitted to our hospital for moderate anemia and mild splenomegalyand was diagnosed with common B-acute lymphatic leukemia(B-ALL) based on laboratory examinations. Peripheral blood examination indicated a white blood cell(WBC) count of $2.45 \times 10^{9} / \mathrm{L}$,red blood cell(RBC) count of $2.27 \times 10^{12} / \mathrm{L}$, hemoglobin(HGB) of $64 \mathrm{~g} / \mathrm{L}$, and platelet count of $68 \times 10^{9} / \mathrm{L}$. Flow-cytometry analysis revealed that the leukemic cell was strongly positive for CD10; moderately positive for CD34,HLA-DR,CD13,CD33,CD19,TDT, and cCD79a; weakly positive for CD38 and CD22; and negative for CD20,cIgM,sIgM, and MPO.Cytogenetic and molecular testing demonstrated that the leukemic cells were positive for TEL/AML1 fusion gene.

Wright-Giemsa staining of the bone marrow aspiration smear suggested that a few giant pink inclusions were present in the cytoplasm. These inclusions, which were located next to the nucleus, exhibited differences in size and appearance upon light microscopic observation(Figs.1A,1B,1C, and 1D). There was marked heterogeneity among the granules.Some of them were translucent, while others were dark. Moreover, the inclusions exhibited slight reactivity in PAS staining(Figs. $1 \mathrm{E}$ and $1 \mathrm{~F}$ ). Cytochemical examination indicated negative expression for MPO.

Transmission electron microscopy (TEM) showed that most of the giant inclusions were located in the perinuclear region, which contained a well-defined membrane. Glycogenosomes of various sizes were present inside giant inclusions, and several multiple-membrane vacuous structures were observed (Figs.2A,2B, 2C,2D,2E, and $2 \mathrm{~F}$ ). The glycogenosomeswere not distributed homogeneously.

\section{Discussion}

The PCH granules are found in many types of cells, including immature myeloid cells, lymphocytes, and monocytes.Gavillet ${ }^{9}$ et al. reported an elderly patient with the presence of $\mathrm{PCH}$ granules after relapse of diagnosed with B-ALL. The expression of CD20 was lost upon comparison with the primary diagnosis. The existence of a connection between the recurrent disease and the missing expression of CD20 is yet to be established.In our study, we first found a novel morphological entity of lymphoblastic cells through TEM. There were $<30 \%(27.7 \%)$ aberrant cells among the total nucleated cells. These anomalous lymphoblasts positively expressed CD10 but not CD20, which is consistent with the case report of Agrawalet al ${ }^{7}$. Nevertheless, the complete blood count (CBC)results signified that the main blood componentswere present at below-normal levels, which differed from the findings in common leukemia.Interestingly, the phenomenon of leucopenia could be found in leukemia, accompanied by anemia or thrombocytopenia ${ }^{10,11}$.

The PCH granules resemble the inclusions noted in CHS, which is a heritable disease linked to the lysosome.The patients with CHS frequently face severe hematologic abnormalities, including hemorrhagic tendency and bacterial infections. The disease is also accompanied by nervous system disorder. Some individuals are initially found to have symptoms of albinism. The Chediak-Higashi syndrome(CHS1)/lysosomal trafficking regulator(LYST) gene was found to be mutated in the disease ${ }^{12}$.Subsequently, the functioning of the lysosomal trafficking protein was compromised by the defective gene, and non-functional enlarged lysosomes were formed in eosinophils,neutrophils, and other granulocytes in the body ${ }^{13}$. Abnormal intracytoplasmic granules, which are generated by a complex process of granular fusion, cytoplasmic injury, and phagocytosis at the moment of secretory granule maturity, were detected in the leukocytes of both peripheral blood and bone marrow ${ }^{14,15}$. The PCH granules were taken the eosinophilic feature in the cytoplasm ${ }^{8}$.Ultrastructural research on $\mathrm{PCH}$ has demonstrated the characteristics of $\mathrm{PCH}$ in acute myeloid leukemia. Similar to the case reported by Pallavi et al. ${ }^{7}$, these eosinophilic inclusions were positive for PAS but negative for MPO in our study. At the same time, a well-defined irregular membranous structure, which contained a variable number and volume of particles, was positioned next to the nucleus. These particles exhibited a high electron density. Some reports have suggested that $\mathrm{PCH}$ is a fusion of abnormal peroxidase-positive small particles ${ }^{7}$. On the contrary, Tulliez ${ }^{16}$ et al. demonstrated that the abnormal granules result from the integration of azurophilic granules in acute myeloid leukemia. However, the PCH particles observed under TEM were composed of membrane-encapsulated scattered glycogen particles. Some of them contained abnormal structures that were 
surrounded by membranes but without any contents, which is different from the CHS granules. The exact mechanism of formation of this abnormal structure is unclear.

In case of B-ALL,the process of B-cell differentiation is arrested. Consequently, the normal hematopoietic process is inhibited due to the increasing number of blast cells. The fusion of TEL-AML1 is common in case of ALL in children and serves as a common leukemogenic marker with favorable outcomes ${ }^{17}$. The TEL-AML1 fusion occursin utero during the embryonic development stage. In other words, it could be detected before the clinical symptoms become visible ${ }^{18}$. The pathogenic gene acting on hematopoietic stem cells (HSC) progresses in the presence of other risk factors. The fusion protein alters the activation of the IFN $\alpha / \beta$ pathway and affects the differentiation and self-renewal abilities of the hematopoietic progenitor cells $(\mathrm{HPC})^{19}$.Ultimately, multiple signaling pathways are activated, thereby promoting the progression of B-ALL ${ }^{20}$. Hatem et al. ${ }^{20}$ demonstrated that the fusion of TEL-AML1 could give rise to the blocked differentiation of B cell.TEL/AML1 fusion is correlated witha good response to chemotherapy,leading to a high survival rate.In the present case, the TEL/AML1 fusion gene was detectedin ALL blasts. There is a possibility that the abnormal blasts failed to differentiate into the next stage but proliferated rapidly. The immune function might have been disrupted owing to the existence of these non-effective lymphocytes. Consequently, the abnormal lymphoblasts containing PCH granules may be a manifestation of TEL/AML1 gene fusion.

Herein, we have reported a special ultrastructural manifestation of PCH in the case of B-ALL.Thefindings testifiythat the entity of $\mathrm{PCH}$ is a glycogenosome although the exact mechanism of its formation remains to be clarified.

\section{Conflict of Interest}

The authors declare that there is no conflict of interest.

\section{Acknowledgments}

This research did not receive any special grant from funding agencies in the public, commercial, or not-forprofit sectors.

\section{References}

1. Daneshbod Y, Medeiros LJ. Pseudo Chediak-Higashi anomaly in acute monoblastic leukemia. Blood . Nov 24 2016;128(21):2583. doi:10.1182/blood-2016-08-732339

2. Aruga Y, Arakawa A, Ono K, Ogawa C, Matsushita H. Pseudo-Chediak-Higashi granules and Auer rods in mixed phenotype acute leukaemia, T/myeloid, not otherwise specified. Br J Haematol . Jan 2018;180(2):175. doi:10.1111/bjh.14924

3. Gajendra S, Gupta R, Sharma A, Gupta R, Gogia A. Acute myeloid leukaemia with Pseudo-ChediakHigashi granules and intracytoplasmic vacuoles. Eur J Haematol . Jun 16 2011;doi:10.1111/j.16000609.2011.01668.x

4. Vargas MT, Escamilla V, Morales-Camacho RM, et al. Multiple pseudo-Chediak-Higashi inclusions associated with MYC deletion in a patient with acute myeloid leukemia. Acta Haematol . 2015;133(4):3213. doi:10.1159/000366095

5. Tsai IM, Tsai CC, Ladd DJ. Pseudo-Chediak-higashi anomaly in chronic myelogenous leukemia with myelofibrosis. Am J Clin Pathol . Jun 1977;67(6):608-9. doi:10.1093/ajcp/67.6.608

6. Avci Z, Malbora B, Ozbek N. Pseudo chediak-higashi anomaly. Turk J Haematol . Jun 2013;30(2):230-1. doi:10.4274/Tjh.2011.0010

7. Agrawal P, Kumar N, Sharma P, Varma S, Varma N. Pseudo chediak-higashi granules in acute lymphoblastic leukemia: a rare entity.Indian J Hematol Blood Transfus . Sep 2014;30(3):201-3. doi:10.1007/s12288012-0206-3 
8. Pradhan S, Chauhan S. Pseudo-Chediak-Higashi inclusions in a case of acute lymphoblastic leukemia. Blood Res . Dec 2017;52(4):240. doi:10.5045/br.2017.52.4.240

9. Gavillet M, Spertini O, Blum S. Pseudo Chediak-Higashi anomaly in blasts from acute lymphoblastic leukemia. Leuk Res . Jul 2018;70:87-90. doi:10.1016/j.leukres.2018.06.004

10. Ziemann F, Metzeler K. [Incidental finding of leukopenia - directly to the hematologist?]. MMW Fortschr Med . Apr 2020;162(7):38-41. Zufallsbefund Leukopenie - unverzuglich zum Hamatologen? : Onkologische Zufallsbefunde. doi:10.1007/s15006-020-0389-8

11. Chiravuri S, De Jesus O. Pancytopenia. StatPearls . 2021.

12. Kaplan J, De Domenico I, Ward DM. Chediak-Higashi syndrome.Curr Opin Hematol . Jan 2008;15(1):229. doi:10.1097/MOH.0b013e3282f2bcce

13. Maaloul I, Talmoudi J, Chabchoub I, et al. Chediak-Higashi syndrome presenting in accelerated phase: A case report and literature review.Hematol Oncol Stem Cell Ther . Jun 2016;9(2):71-5. doi:10.1016/j.hemonc.2015.07.002

14. Ajitkumar A, Yarrarapu SNS, Ramphul K. Chediak Higashi Syndrome.StatPearls . 2021.

15. Barak Y, Nir E. Chediak-Higashi syndrome. Am J Pediatr Hematol Oncol . Spring 1987;9(1):42-55. doi:10.1097/00043426-198721000-00008

16. Tulliez M, Vernant JP, Breton-Gorius J, Imbert M, Sultan C. Pseudo-Chediak-Higashi anomaly in a case of acute myeloid leukemia: electron microscopic studies. Blood . Oct 1979;54(4):863-71.

17. Kempski HM, Sturt NT. The TEL-AML1 fusion accompanied by loss of the untranslocated TEL allele in B-precursor acute lymphoblastic leukaemia of childhood. Leuk Lymphoma . Dec 2000;40(1-2):39-47. doi:10.3109/10428190009054879

18. Schindler JW, Van Buren D, Foudi A, et al. TEL-AML1 corrupts hematopoietic stem cells to persist in the bone marrow and initiate leukemia. Cell Stem Cell . Jul 2 2009;5(1):43-53. doi:10.1016/j.stem.2009.04.019

19. de Laurentiis A, Hiscott J, Alcalay M. The TEL-AML1 fusion protein of acute lymphoblastic leukemia modulates IRF3 activity during early B-cell differentiation. Oncogene . Dec 3 2015;34(49):6018-28. doi:10.1038/onc.2015.50

20. Sabaawy HE, Azuma M, Embree LJ, Tsai HJ, Starost MF, Hickstein DD. TEL-AML1 transgenic zebrafish model of precursor B cell acute lymphoblastic leukemia. Proc Natl Acad Sci U S A . Oct 10 2006;103(41):15166-71. doi:10.1073/pnas.0603349103

\section{Figure legend}

Figure 1. The morphology and PAS staining of giant inclusions in lymphoblastic cells.

A, B, C, D. Giemsa-Wright's staining of patient's bone marrow smear

The inclusions are located next to the nucleus, showing heterogeneous coloring.

E, F. PAS staining of lymphoblast in the bone marrow. The inclusions are mildly positive in lymphoblastic cells (as indicated by the arrow).

Figure 2. PCH granules by TEM

A. The lymphoblast with PCH granules located near the nucleus. PCH consists of a membrane-enclosing structure within glycogen granules.

B. The enlarged view of a rectangular area of Figure A. The PCH granules are composed of compact glycogen granules. 
C. The different morphology of PCH granules indicated in the rectangular area.

D. The enlarged view of the rectangular area of Figure C. In addition to the sparsely distributed glycogen granules, three multiple-membrane vacuous structures can be seen.

E, F. Different PCH inclusions in patient's lymphoblastic cells (as indicated by the arrow).

\section{Hosted file}

Title page.docx available at https://authorea.com/users/435624/articles/538516-ultrastrutalinvestigation-on-pseudo-chediak-higashi-abnormality-in-acute-lymphoblastic-leukemia-acase-report
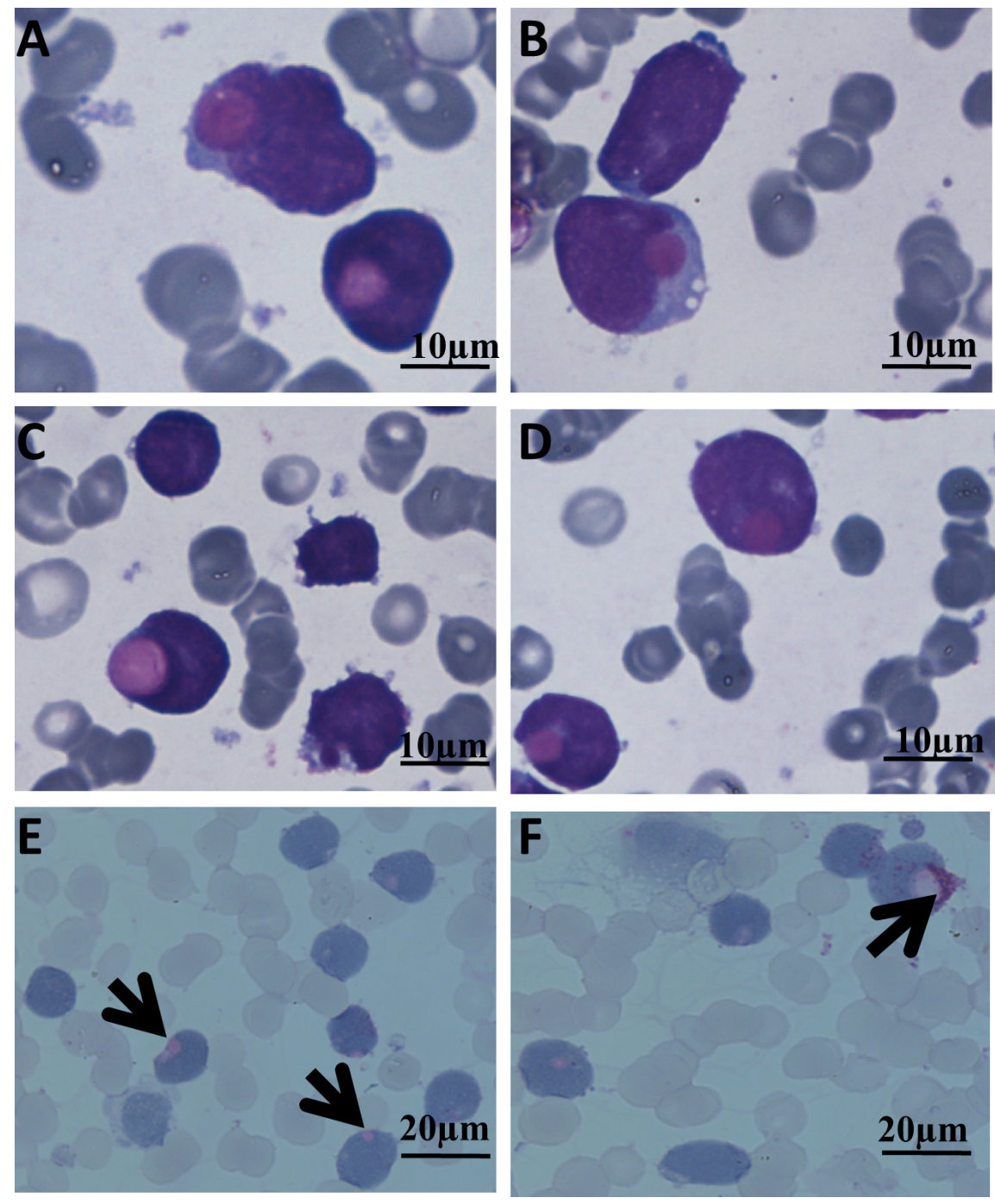

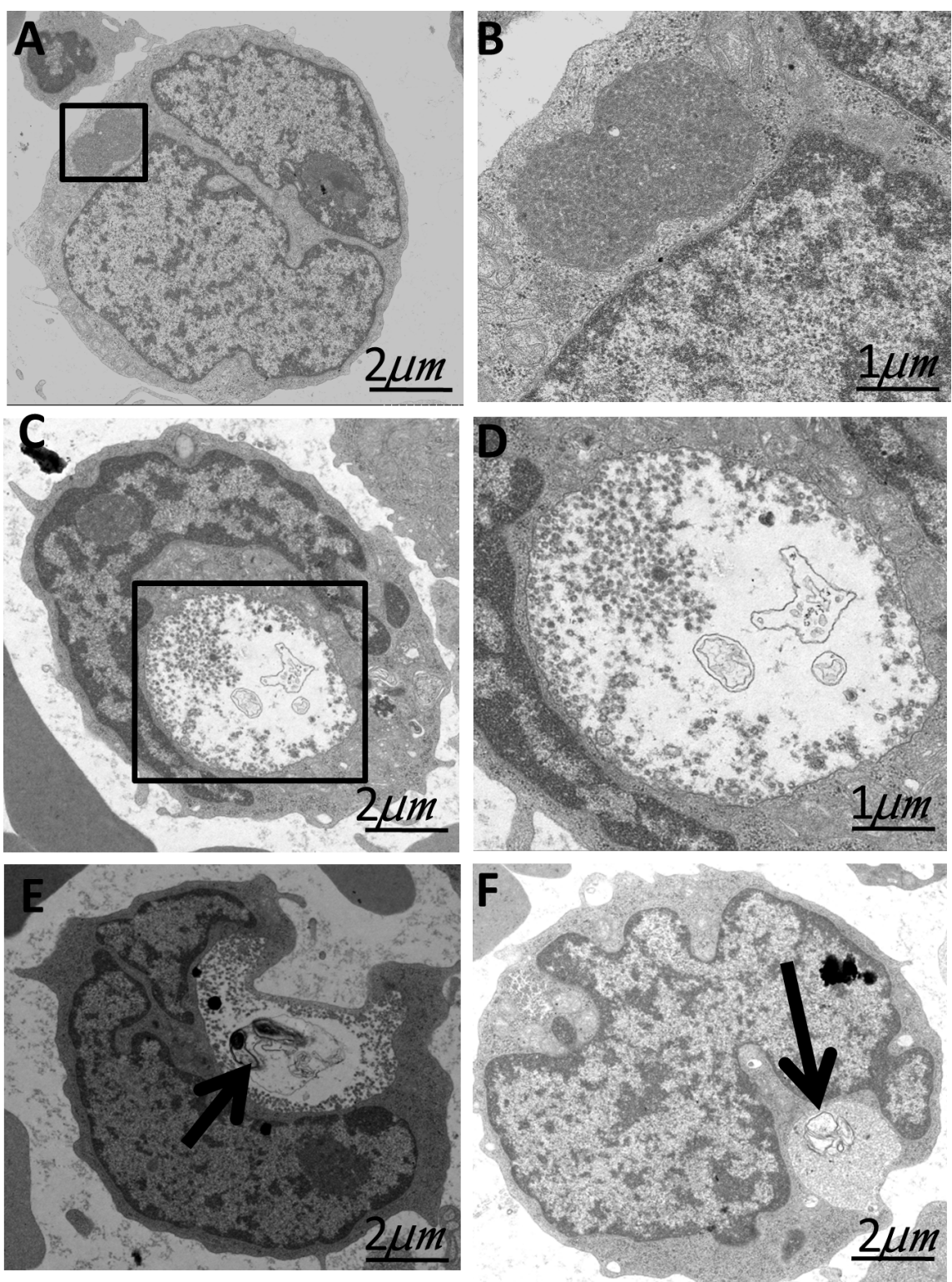